\title{
Low rates of patients meeting return to sport criteria 9 months after anterior cruciate ligament reconstruction: a prospective longitudinal study
}

\author{
Wouter Welling ${ }^{1,2} \cdot$ Anne Benjaminse $^{2,3} \cdot$ Romain Seil $^{4,5} \cdot$ Koen Lemmink $^{2} \cdot$ Stefano Zaffagnini $^{6} \cdot$ Alli Gokeler $^{2}$
}

Received: 22 December 2017 / Accepted: 20 March 2018 / Published online: 24 March 2018

(c) The Author(s) 2018

\begin{abstract}
Purpose The purpose of the current prospective study was to assess the changes over time in patients tested at 6 months and 9 months after anterior cruciate ligament reconstruction (ACLR) with a return to sport (RTS) test battery. It was hypothesized that more patients passed RTS criteria at 9 months compared to 6 months.

Methods Sixty-two ACLR patients performed a test battery at an average of $6.5 \pm 0.7$ and $9.5 \pm 0.9$ months after ACLR. All patients underwent a standardized rehabilitation protocol. The test battery consisted of the following tests: a jump-landing task assessed with the Landing Error Scoring System (LESS), three single-leg hop tasks (single-leg hop test, triple-leg hop test, side hop test), isokinetic quadriceps and hamstring strength at 60, 180 and $300 \% \mathrm{~s}$ and two questionnaires (IKDC and ACL-RSI). Cut off criteria were set as Limb Symmetry Index (LSI) $>90 \%$ (for isokinetic strength and for single-leg hop tasks), LESS $<5$, IKDC score within 15th percentage of healthy subjects and ACL-RSI $>56$ respectively.

Results At 6 months, two patients (3.2\%) passed all criteria. At 9 months, seven patients (11.3\%) passed all criteria. Patients improved in all RTS criteria over time except for the IKDC score. Twenty-nine patients (46.8\%) did not pass the strength criterion at $60^{\circ}$ s at 9 months after ACLR.

Conclusions The percentages of patients passing all RTS criteria were low at both 6 and 9 months after ACLR. Quadriceps strength revealed persistent deficits and the lack of improvement in the IKDC score questionnaires shows insufficient selfreported knee function for RTS.
\end{abstract}

Level of evidence III.

Keywords Anterior cruciate ligament $\cdot$ Return to sport $\cdot$ Strength $\cdot$ Hop tests $\cdot$ Movement analysis

Wouter Welling

wouter.welling@mcz.nl

1 Medisch Centrum Zuid, Sportlaan 2-1, 9728 PH Groningen, The Netherlands

2 Center for Human Movement Science, University of Groningen, University Medical Center Groningen, Antonius Deusinglaan 1, 9713 AV Groningen, The Netherlands

3 School of Sport Studies, Hanze University Groningen, Zernikeplein 17, 9747 AS Groningen, The Netherlands

4 Département de l'Appareil Locomoteur, Centre Hospitalier de Luxemburg, 4 Rue Nicolas Ernest Barblé, 1210 Luxembourg, Luxembourg

5 Sports Medicine Research Laboratory, Luxembourg Institute of Health, 4 Rue Nicolas Ernest Barblé, 1210 Luxembourg, Luxembourg

6 Rizzoli Orthopaedic Institute, University of Bologna, Via Gulio Cesare Pupilli 1, 40135 Bologna, Italy

\section{Introduction}

The aim for most athletes who undergo an anterior cruciate ligament reconstruction (ACLR) is to restore full knee stability and functional capacity allowing them to return to sport (RTS) $[14,15]$. The decision for RTS after ACLR is one of the most challenging and difficult decisions for clinicians to make [45]. The patient expectations for RTS are high, since $94 \%$ expect to return to pre-injury level of sports [16]. However, current RTS rates to competitive sports are relatively low $(55 \%)[2,5]$ and the overall re-injury rate after ACLR ranges between 15 and $23 \%$ for young athletes ( $<25$ years) [43]. One major reason for these percentages may be the failure to obtain sufficient knee function and muscle strength, which are suggested to be critical for RTS $[36,37]$. 
Traditionally, RTS is recommended after 6 months. However, this timeframe has been questioned in the literature [2, $14,18]$, since the risk of sustaining an ACL re-injury is the highest during the early period of RTS (6-12 months) [21, 26]. To decrease the re-injury risk, it is advised to delay RTS to at least 9 months after ACLR. The most commonly used assessments described in literature for RTS decision making after ACLR are strength testing, performance-based functional testing (e.g. hop tests) and self-reported knee function [39]. For strength tests and hop tests, limb symmetry index (LSI) values are commonly used to calculate the difference in score between the non-injured and injured leg. LSIs of $>90 \%$ are commonly considered as cut off values for RTS $[1,20,31]$

RTS after ACLR is complex in nature, and it is suggested to use multifactorial test batteries to determine to readiness for RTS of an athlete [18]. It is unclear how ACLR patients progress on multifactorial RTS criteria over time. Therefore, the purpose of the current prospective study was to assess the changes over time in patients tested at 6 and 9 months after ACLR with a RTS test battery. It was hypothesized that more patients passed RTS criteria at 9 months compared to 6 months.

\section{Materials and methods}

Patients were prospectively recruited by the same researcher (W.W.) during their rehabilitation in an outpatient physical therapy clinic. Data collection took place between 2015 and 2017. 81 ALCR patients fulfilled inclusion criteria (Fig. 1). Six patients stopped their rehabilitation before 6 months due to a lack of time for rehabilitation (4) or pregnancy (2). Additionally, 13 patients stopped their rehabilitation before 9 months due to lack of motivation (5), a lack of time (3), moving (3) or a missed appointment for the test session (2). Therefore, 62 patients (mean age $24.2 \pm 6.2$ years) were included. Detailed demographics are presented in Table 1. Inclusion criteria for the patients and the standardized rehabilitation protocol have been described in detail earlier [18]. All patients performed a test battery two times, at an average of $6.5 \pm 0.7$ and $9.5 \pm 0.9$ months after ACLR.

\section{Procedures}

All subjects were tested by the same researcher (W.W.). The test battery used in the current study included the following tests in this order [18]: a jump-landing task assessed with the Landing Error Scoring System (LESS), single-leg hop test (SLH), triple-leg hop test (TLH), side hop test (SH), isokinetic strength testing for quadriceps and hamstring strength at a velocity of 60,180 and $300^{\circ} / \mathrm{s}$ with $5,10,10$ maximal concentric repetitions for flexion and extension.

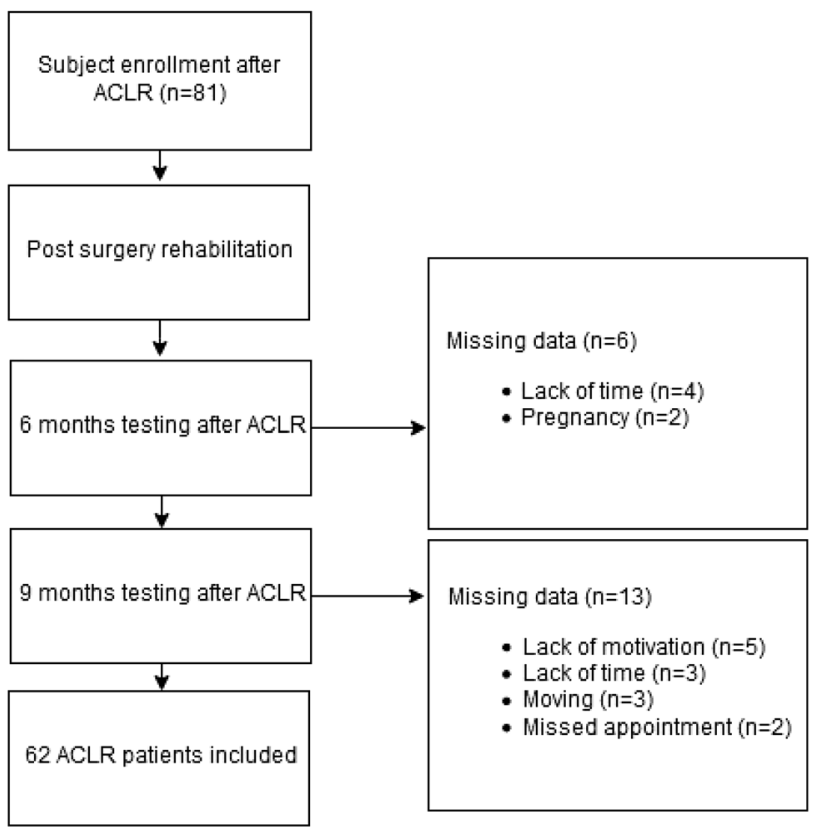

Fig. 1 Flow chart of the time line during data analysis. ACLR anterior cruciate ligament reconstruction

After testing, two patient questionnaires were completed by every patient: the International Knee Documentation Committee Subjective Knee Form (IKDC) [27] and the Anterior Cruciate Ligament-Return to Sport after Injury Scale (ACL-RSI) [3].

\section{Data reduction}

All tests used in the test battery have shown to be highly reliable (LESS: $\mathrm{ICC}=0.91$; SLH: $\mathrm{ICC}=0.97$; TLH: ICC $=0.80-0.92$; SH: ICC $=0.84-0.96$; isokinetic device: ICC $=0.91-0.99) \quad[24,29,33,38]$. The LESS was analyzed by playing frontal and sagittal videos frame by frame [32]. Muscular strength was tested with an isokinetic device (Biodex System 3; Biodex Medical Systems, Inc, Shirley, NY). For the isokinetic quadriceps and hamstring strength and single-leg hop tasks, LSI values were calculated. Absolute values were normalized to bodyweight $(\mathrm{BW})$ for the isokinetic quadriceps peak torque test at $60 \%$ for the injured leg. The recommended threshold has been set at $>3.0 \mathrm{Nm} / \mathrm{kg}$ [25]. Additionally, hamstring/quadriceps (H/Q) ratios were calculated at $300 \%$ for the injured leg with a recommended cutoff set at $>55 \%$ for females and $>62.5 \%$ for males [22]. For the IKDC, the 15th percentile from uninjured athletes was chosen as the cutoff score [27]. Additionally, for the ACL-RSI a cut off score of 56 points was recommended [3]. The study protocol was approved by the Medical Ethical Committee (ID 2012.362) of the University of Groningen, 
Table 1 Demographic data included ACLR patients

\begin{tabular}{llll}
\hline & All $(n=62)$ & Males $(n=45)$ & Females $(n=17)$ \\
\hline Age (years) & $24.2 \pm 6.2$ & $25.3 \pm 6.3$ & $21.2 \pm 5.0$ \\
Mass (kg) & $75.8 \pm 11.1$ & $78.6 \pm 10.3$ & $68.4 \pm 10.1$ \\
Type of graft $(n)$ & HT(36), PT(25), AG(1) & HT(21), PT(23), AG(1) & HT(15), PT(2) \\
Time post-surgery first-test moment (months) & $6.5 \pm 0.7$ & $6.5 \pm 0.7$ & $6.4 \pm 0.6$ \\
Time post-surgery second-test moment (months) & $9.5 \pm 0.9$ & $9.5 \pm 0.9$ & $9.4 \pm 0.7$ \\
Number of therapy sessions first-test moment & $52.7 \pm 15.6$ & $53.5 \pm 12.1$ & $50.7 \pm 22.7$ \\
Number of therapy sessions second-test moment & $74.3 \pm 20.1$ & $76.0 \pm 18.6$ & $69.7 \pm 23.6$ \\
Sport & $\mathrm{F}(45), \mathrm{B}(6), \mathrm{H}(4), \mathrm{T}(3), \mathrm{K}(2)$, & $\mathrm{F}(36), \mathrm{B}(5), \mathrm{T}(2), \mathrm{K}(1), \mathrm{R}(1)$ & $\mathrm{F}(9), \mathrm{H}(4), \mathrm{B}(1)$, \\
& $\mathrm{R}(1), \mathrm{V}(1)$ & & $\mathrm{K}(1), \mathrm{T}(1)$, \\
& & & $\mathrm{V}(1)$ \\
\hline
\end{tabular}

$K g$ kilogram, $H T$ hamstring tendon graft, $P T$ bone-patellar tendon graft, $A G$ allograft, $F$ football, $B$ basketball, $H$ handball, $T$ tennis, $K$ korfball, $R$ rugby, $V$ volleyball

and informed consent was obtained from all patients prior to data collection.

\section{Statistical analysis}

All data were normally distributed as analyzed with SPSS version 20 (SPSS 244 Inc, Chicago, IL). To determine differences between time (6 and 9 months) and legs (noninjured leg and injured leg), a $2 \times 2$ ANOVA was conducted. Additionally, a $2 \times 2$ ANOVA was conducted to determine difference between patients with a hamstring tendon graft (HT) and patients with a bone-patellar tendon graft (PT). A power analysis ( $\mathrm{G}^{*}$ Power, version 3.1 .7$)$ was used to calculate the required sample size. With an effect size of 0.50 (medium-large effect ANOVA) and an alpha of 0.05, 27 subjects were required to obtain a power of 0.80 [11].

A regression analysis was used to evaluate whether specific RTS criteria (independent variable) can predict passing/failing the total test battery (dependent variable). Also, the regression analysis was used to evaluate the proportion that each variable can predict the outcome of interest. Participants' sex and age were used as covariates in the regression analysis. The forward selection method of the regression analysis was used to determine significant predictor variables. Only significant predictors were entered into the regression analysis. Statistical significance was set at $p<0.05$ level of confidence.

\section{Results}

Of the 62 included patients, 2 patients $(3.2 \%)$ passed all RTS criteria at 6 months and 7 patients $(11.3 \%)$ at 9 months. Five patients $(8.1 \%)$ passed the strength test criteria at 6 months and 13 patients $(21.0 \%)$ at 9 months. 39 patients $(62.9 \%)$ passed all hop tests at 6 months and 48 patients $(77.4 \%)$ at 9 months. An overview of the results can be found in Tables 2, 3 and 4 and Figs. 2 and 3.

6 months after ACLR, the mean IKDC score was $81.1 \pm 7.8$ with 36 patients $(58.1 \%)$ classified as having self-reported knee function with normal ranges and 9 months after ACLR, the mean IKDC score was 81.7 \pm 6.9 and 39 patients $(62.9 \%)$ were classified as having selfreported knee function with normal ranges. For the ACL-RSI, the mean score was $61.7 \pm 16.6$ at 6 months and 37 patients $(59.7 \%)$ passed the criteria. At 9 months, the mean ACL-RSI score was $67.3 \pm 18.1$ and 45 patients (72.6\%) passed the criteria.

Significant higher IKDC score $(p=0.001)$ and LSI TLH ( $p=0.017$ ) were found in patients with a HT graft are compared to patients with a PT graft at 6 months. At 9 months, patients with a HT graft had significant higher LSI quadriceps peak torque at $60^{\circ} / \mathrm{s}(p=0.036)$ and higher LSI SH $(p=0.043)$ compared to patients with a PT graft.

\section{Discussion}

The main finding of the current study is that only $3.2 \%$ of the patients passed all RTS criteria at 6 months after ACLR. Furthermore, only $11.3 \%$ of the patients passed all RTS criteria at 9 months after ACLR. The results show a lack of improvement in quadriceps strength and self-reported knee function at 9 months after ACLR. The percentage of patients that passed RTS criteria in the current study after 6 months is similar to the previous results of Gokeler et al. [18]. After 9 months, only $11.3 \%$ of the patients in the current study passed the RTS criteria. These findings are in agreement with Toole et al. [39], showed that $13.9 \%$ of patients passed RTS criteria (8.2 \pm 2.4 months after ACLR).

Almost half of the patients $(46.8 \%)$ did not pass the quadriceps strength criterion (LSI $>90 \%$ ) at $60 \%$ s at 9 months after ACLR. Similarly, only $40.3 \%$ of the patients 
Table 2 Pass criteria and percentage of patients that passed specific criterion at 6 months and 9 months

\begin{tabular}{lll}
\hline Pass criteria and percentage of patients that passed criterion & 6 months & 9 months \\
\hline LSI $>90 \%$ peak torque quadriceps $60 \% \mathrm{~s}$ & 33.9 & 53.2 \\
LSI $>90 \%$ peak torque hamstrings $60 \% \mathrm{~s}$ & 67.7 & 74.2 \\
LSI $>90 \%$ peak torque quadriceps $180 \% \mathrm{~s}$ & 43.5 & 56.5 \\
LSI $>90 \%$ peak torque hamstrings $180 \% \mathrm{~s}$ & 75.8 & 72.6 \\
LSI $>90 \%$ peak torque quadriceps $300 \% \mathrm{~s}$ & 38.7 & 59.7 \\
LSI $>90 \%$ peak torque hamstrings $300 \% \mathrm{~s}$ & 80.6 & 85.5 \\
Peak torque $>3.0$ Nm/kg for the injured leg at $60 \%$ s normalized to BW & 27.4 & 40.3 \\
H/Q ratio $>55 \%$ for females and $>62.5 \%$ for males for the injured leg at $300 \% / \mathrm{s}$ & 90.3 & 91.9 \\
LSI $>90 \%$ single-leg hop test & 74.2 & 96.8 \\
LSI $>90 \%$ triple-leg hop test & 75.8 & 93.5 \\
LSI $>90 \%$ side hop test & 45.2 & 83.9 \\
LESS $<5$ & 51.6 & 80.6 \\
IKDC score within $15 \%$ of healthy gender-age-matched subjects & 58.1 & 62.9 \\
ACL-RSI $>56$ & 59.7 & 72.6 \\
\hline
\end{tabular}

LSI limb symmetry index, ${ }^{\circ}$ degrees, $s$ seconds, $\mathrm{Nm}$ newton metre, $\mathrm{kg}$ kilogram, $H / Q$ hamstring/quadriceps, LESS Landing Error Scoring System, IKDC International Knee Documentation Committee Subjective Knee Form, $A C L-R S I$ Anterior Cruciate Ligament-Return to Sport after Injury Scale passed the criterion $>3.0 \mathrm{Nm} / \mathrm{kg}$ for the injured leg at $60^{\circ} / \mathrm{s}$. The absolute increase in quadriceps strength between 6 and 9 months was $23.0 \mathrm{Nm}$, which was lower than the minimal detectable change (MDC) of $33.9 \mathrm{Nm}$ [23]. This indicates a lack of clinical improvement in quadriceps strength from 6 to 9 months after ACLR. Of concern is that these findings are in line with previous research at 6 months after ACLR [18] and, therefore, it is suggested to change the standardized training protocol. Quadriceps strength has been suggested to be essential after ACLR since greater quadriceps muscle strength is a factor associated with successful RTS after ACLR [12]. Furthermore, the results of the current study show that quadriceps strength at $60 \%$ s (only at 9 months after ACLR), 180 and $300 \%$ (at 6 and 9 months after ACLR) are significant predictors whether patients will pass all RTS criteria. These findings highlight the importance of symmetric quadriceps strength after ACLR.

39 patients (62.9\%) passed all hop test criteria at 6 months and 48 patients (77.4\%) at 9 months. In addition, patients scored significantly better on all three hop tests at 9 months compared to 6 months after ACLR. The absolute increase in jumping distance between 6 and 9 months on SLH was $10.6 \%(16.0 \mathrm{~cm})$, which was higher than the MDC of $8.1 \%$ [40]. In addition, the absolute increase in jumping distance between 6 and 9 months on the TLH was $10.8 \%(51.7 \mathrm{~cm})$, which was higher than the MDC of $10.0 \%$ [40]. The LSI values of all three hop tests were significantly better at 9 months compared to 6 months after ACLR. The use of LSI is a common method to calculate the score between the injured and non-injured leg [1, 20, 31]. However, caution is warranted for the use of LSI since this method can mask bilateral deficits since the non-injured leg can also be affected by the injury and inactivity time [17]. The use of normative data is suggested to be a more adequate method when analyzing patient data. A comparison with normative data [30] shows that especially our male patients do not meet the jump distance at 9 months after ACLR in the SLH $(192.0 \mathrm{~cm}$ [30] vs. $175.4 \mathrm{~cm}$ for the injured leg) and in the TLH $(632.0 \mathrm{~cm}$ [30] vs. $558.5 \mathrm{~cm}$ for the injured leg). These results show that the use of LSI may underestimate performance deficits and should, therefore, be analyzed with caution when used as a criterion for RTS after ACLR [17, 42].

There is a lack of clinical improvement on the IKDC between 6 and 9 months after ACLR. The absolute change in IKDC score between 6 and 9 months was 0.6 , which was lower than the MDC of 8.8 [19]. This indicates insufficient self-reported knee function at 9 months after ACLR. These findings are of concern, since significant lower IKDC scores were found in patients who did not RTS after ACLR [4]. Additionally, the results of the current study show that the ACL-RSI (at 6 and 9 months after ACLR) and the IKDC (at 9 months after ACLR) are significant predictors of passing all RTS criteria in our study. Patients with a PT graft had lower IKDC scores compared to patients with a HT graft at 6 months. Patients did improve their LESS score over time. However, at 9 months after ACLR, still $19.4 \%$ of the patients did not pass the LESS $<5$ criterion. In more detail, $8.1 \%$ of the patients showed a LESS score $>6$ (poor jump landing biomechanics) [13, 18, 33]. Furthermore, the LESS is a significant predictor for patients passing all RTS criteria at both 6 and 9 months after ACLR. Therefore, it is recommended to add movement analysis in the decision-making process for RTS [14]. Asymmetrical movement patterns (for example increased knee valgus) are suggested to increase 


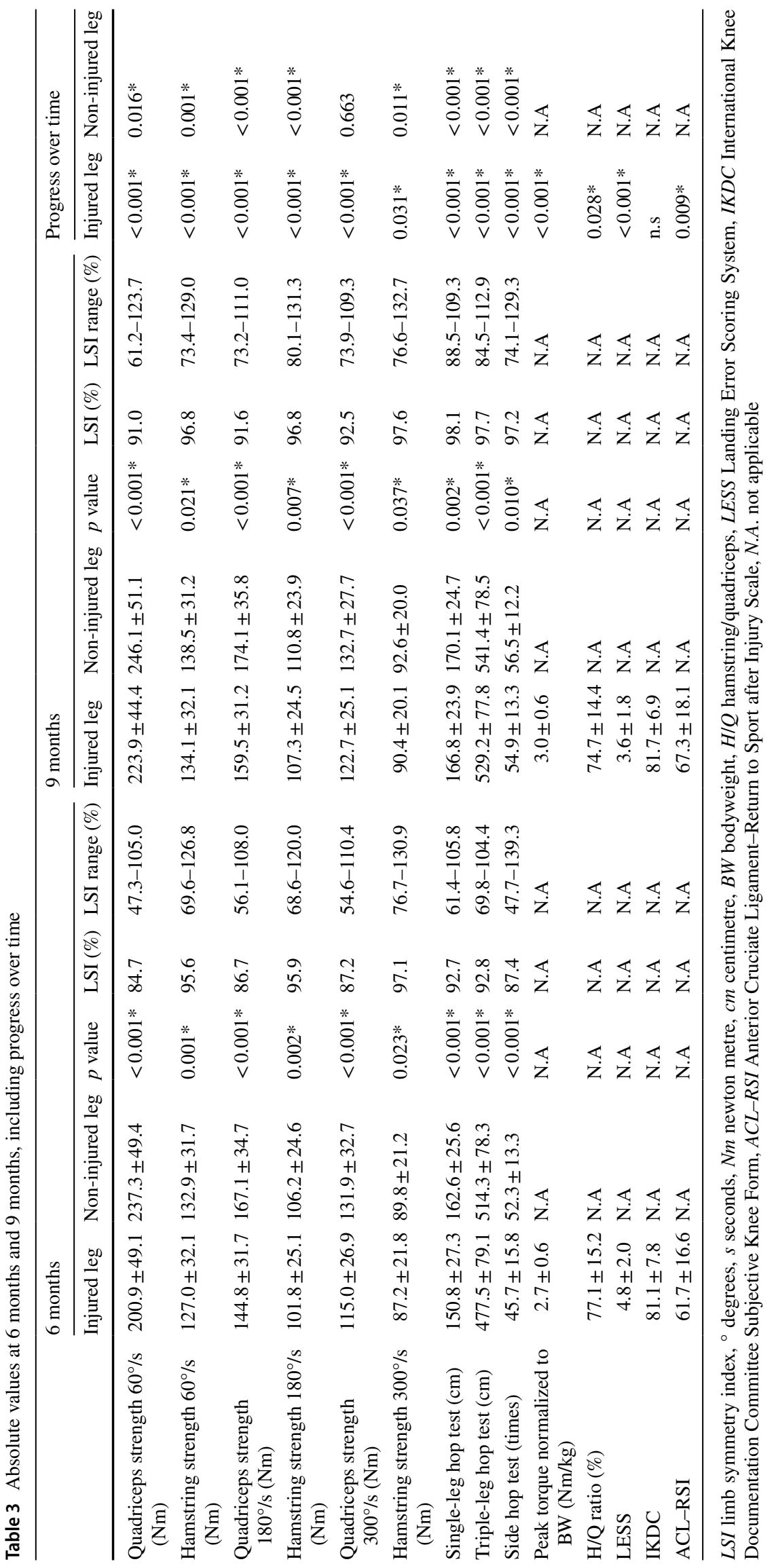


Table 4 Combination of specific RTS criteria for the prediction of passing all RTS criteria at 6 and 9 months after ACLR

\begin{tabular}{llrll}
\hline Dependent variable & Independent variable & \multicolumn{1}{l}{$\beta$} & $p$ value \\
\hline Passing all RTS criteria at 6 months & Intercept & -16.360 & - & - \\
$R^{2}=0.801$ & LSI quadriceps strength at $300^{\circ} / \mathrm{s}$ & 0.052 & 0.179 & $<0.001^{*}$ \\
& Peak torque $>3.0 \mathrm{~N} \mathrm{~m} / \mathrm{kg}$ & 1.255 & 0.268 & $<0.001^{*}$ \\
& LSI hamstring strength at $180^{\circ} / \mathrm{s}$ & 0.067 & 0.234 & $0.002^{*}$ \\
& LESS & -0.317 & -0.217 & $0.003^{*}$ \\
& LSI SH & 0.033 & 0.214 & $0.004^{*}$ \\
Passing all RTS criteria at 9 month & ICL-RSI & 0.031 & 0.179 & $0.026^{*}$ \\
$R^{2}=0.774$ & LSI quadriceps strength at $180^{\circ} / \mathrm{s}$ & 0.076 & 0.264 & $0.034^{*}$ \\
& LSI quadriceps strength at $180^{\circ} / \mathrm{s}$ & 0.037 & 0.118 & $<0.001^{*}$ \\
& LESS & -0.462 & -0.298 & $<0.001^{*}$ \\
& LSI hamstring strength at $180^{\circ} / \mathrm{s}$ & 0.096 & 0.317 & $<0.001^{*}$ \\
& IKDC & 0.108 & 0.270 & $<0.001^{*}$ \\
& ACL-RSI & 0.031 & 0.204 & $0.022^{*}$ \\
& LSI quadriceps strength at $60^{\circ} / \mathrm{s}$ & 0.069 & 0.263 & $0.021^{*}$ \\
& LSI quadriceps strength at $300^{\circ} / \mathrm{s}$ & 0.097 & 0.273 & $0.017^{*}$ \\
\hline
\end{tabular}

${ }^{\circ}$ degrees, $s$ seconds, Nm newton metre, LESS Landing Error Scoring System, SH side hop test, $k g$ kilogram, IKDC International Knee Documentation Committee Subjective Knee Form, $A C L-R S I$ Anterior Cruciate Ligament-Return to Sport after Injury Scale

*Significant predictor

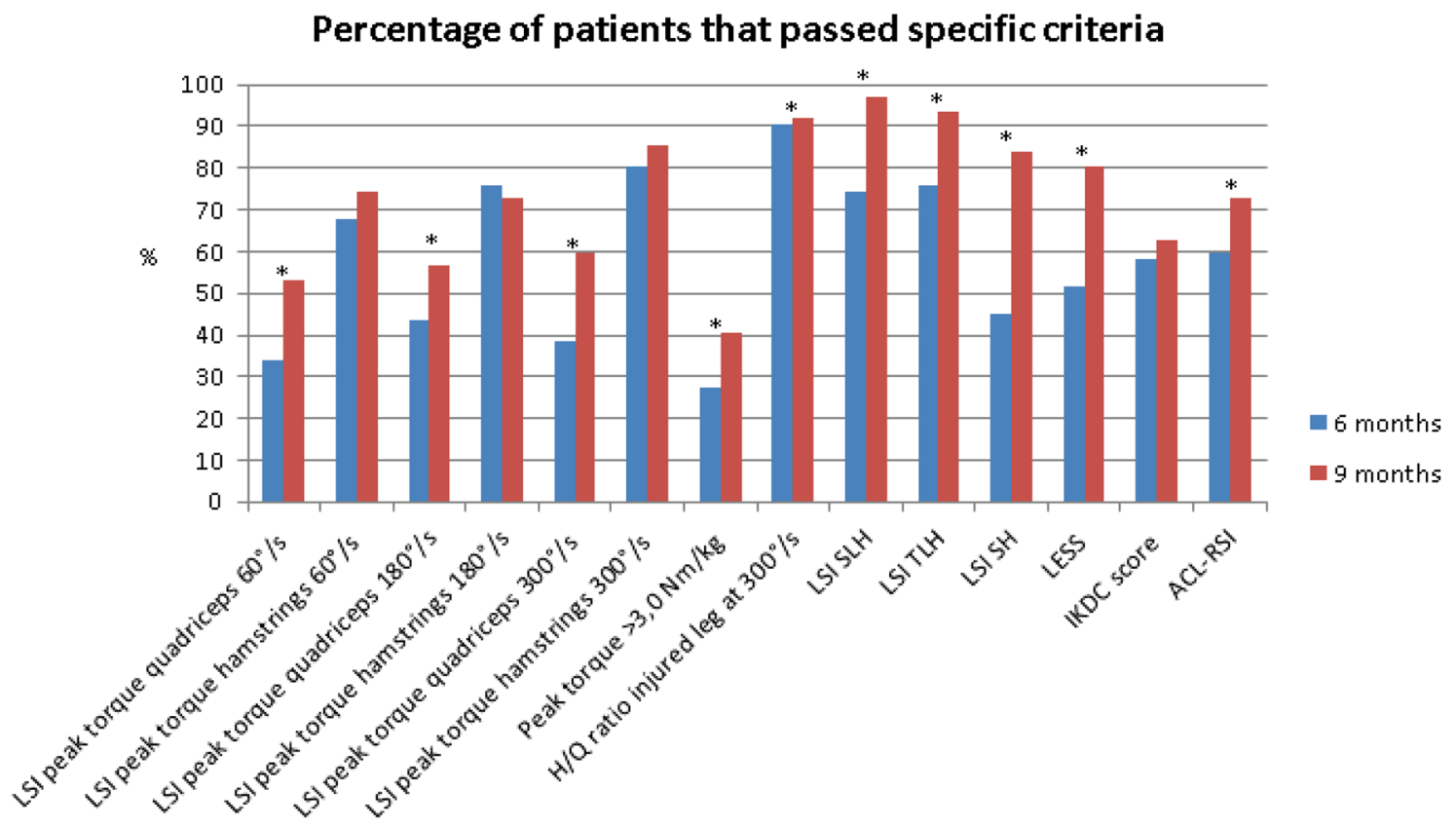

Fig. 2 Overview of percentages of patients that passed specific RTS criteria at 6 and 9 months. LSI limb symmetry index, ${ }^{\circ}$ degrees, $s$ seconds, $\mathrm{Nm}$ newton metre, $\mathrm{kg}$ kilogram, $H / Q$ hamstring/quadriceps, $S L H$ single-leg hop test, TLH triple-leg hop test, $S H$ side hop, LESS

the re-injury risk and should be incorporated in RTS tests $[6,8,34,41,44]$.
Landing Error Scoring System, IKDC International Knee Documentation Committee Subjective Knee Form, $A C L-R S I$ Anterior Cruciate Ligament-Return to Sport after Injury Scale, *significant difference

Between graft comparison showed a higher LSI quadriceps strength at $60 \%$ in patients with a HT graft compared to patients with a PT graft at 9 months. Only $40 \%$ of the PT 
Fig. 3 LSI values for males at 6 months and 9 months for quadriceps strength at $60 \%$, hamstring strength at $60 \%$, single-leg hop test (SLH), triple-leg hop test (TLH) and side hop test (SH). LSI limb symmetry index, $S L H$ single-leg hop test, $T L H$ triple-leg hop test, SH side hop test

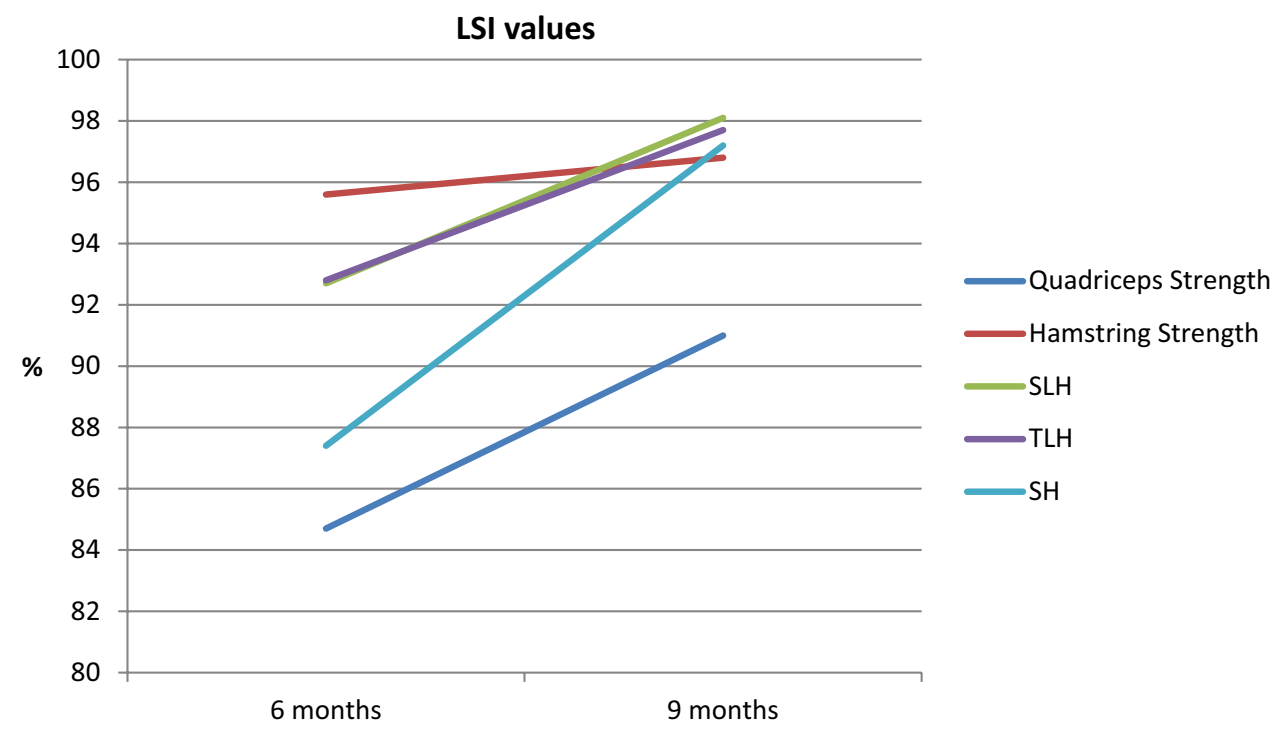

patients passed the LSI $>90 \%$ quadriceps strength at $60 \%$ at 9 months compared to $63.9 \%$ of the HT patients. This is in line with previous research, showing a greater quadriceps deficit in PT patients compared with HT patients at 6 months after ACLR [28].

Since only $11.3 \%$ of the patients passed all RTS criteria, the results of the current study may suggest that training loads were not high enough during rehabilitation. This could lead to unwanted effects when increasing the training load after returning to the field, since an increase in training load could increase the risk for re-injury dramatically [9]. Therefore, acute/chronic workload ratio (workload last week/workload of the last 4 weeks) should be added in the RTS decision [9]. It is suggested that the acute/chronic workload ratio should be increased carefully during the rehabilitation. In addition, in the last part of the rehabilitation it is suggested to add more sport specific training, for example, field training focused on reactive agility, especially during fatigued circumstances [7, 14]. Especially for RTS (performing at the pre-injury level) or return to performance (performing al least at pre-injury level [14]), fatigue can be a risk factor for re-injury since neuromuscular control is altered under fatigued circumstances $[10,35]$. The test battery used in the current study might not be sufficient for the return to performance phase, in which the physical, physiological and psychological demands are much higher compared to RTS.

There are some limitations that should be noticed. The current study gives recommendations in the relevance of the RTS criteria chosen. However, it was not evaluated if the study results pertaining to return to pre-injury level of sports. Prospective studies are needed to determine and evaluate evidence-based RTS criteria. Second, the current study was focused on testing in a closed, clinical environment. Third, in the current study there were dropouts due to a lack of motivation, which could influence the results (attrition bias).

\section{Conclusion}

The percentages of patients passing all RTS criteria were low at both 6 and 9 months after ACLR. The largest improvements were observed in the three hop tests, whilst quadriceps strength revealed persistent deficits. Also, the lack of improvement in the IKDC and ACL-RSI score questionnaires shows insufficient psychological readiness for RTS. Future research should focus on the effects of more progressive quadriceps strength training.

Funding No funding has been received for this study.

\section{Compliance with ethical standards}

Conflict of interest The authors have no conflicts of interest.

Ethical approval Work has been approved by the ethical committees of the University of Groningen.

Informed consent All subjects signed an informed consent form before data collection took place.

Clinical Trial Registry name and registration The University of Groningen (ECB/2014.1.20_1).

Open Access This article is distributed under the terms of the Creative Commons Attribution 4.0 International License (http://creativeco 
mmons.org/licenses/by/4.0/), which permits unrestricted use, distribution, and reproduction in any medium, provided you give appropriate credit to the original author(s) and the source, provide a link to the Creative Commons license, and indicate if changes were made.

\section{References}

1. Abrams GD, Harris JD, Gupta AK, McCormick FM, Bush- Joseph CA, Verma NN, Cole BJ, Bach BR (2014) Functional performance testing after anterior cruciate ligament reconstruction: a systematic review. Orthop J Sports Med 2(1):1-10

2. Ardern CL, Taylor NF, Feller JA, Webster KE (2014) Fifty-five per cent return to competitive sport following anterior cruciate ligament reconstruction surgery: an updated systematic review and meta-analysis including aspects of physical functioning and contextual factors. Br J Sports Med 48(21):1543-1552

3. Ardern CL, Taylor NF, Feller JA, Whitehead TS, Webster KE (2013) Psychological responses matter in returning to preinjury level of sport after anterior cruciate ligament reconstruction surgery. Am J Sports Med 41(7):1549-1558

4. Ardern CL, Taylor NF, Feller JA, Whitehead TS, Webster KE (2015) Sports participation 2 years after anterior cruciate ligament reconstruction in athletes who had not returned to sport at 1 year: a prospective follow-up of physical function and psychological factors in 122 athletes. Am J Sports Med 43(4):848-856

5. Ardern CL, Webster KE, Taylor NF, Feller JA (2011) Return to the preinjury level of competitive sport after anterior cruciate ligament reconstruction surgery: two-thirds of patients have not returned by 12 months after surgery. Am J Sports Med 39(3):538-543

6. Barber-Westin SD, Noyes FR (2011) Factors used to determine return to unrestricted sports activities after anterior cruciate ligament reconstruction. Arthroscopy 27(12):1697-1705

7. Bien DP, Dubuque TJ (2015) Considerations for late stage AC rehabilitation and return to sport to limit re-injury risk and maximize athletic performance. Int J Sports Phys Ther 10(2):256-271

8. Bizzini M, Hancock D, Impellizzeri F (2012) Suggestions from the field for return to sports participation following anterior cruciate ligament reconstruction: soccer. J Orthop Sports Phys Ther 42(4):304-312

9. Blanch P, Gabbett TJ (2016) Has the athlete trained enough to return to play safely? The acute:chronic workload ratio permits clinicians to quantify a player's risk of subsequent injury. Br J Sports Med 50:471-475

10. Borotikar BS, Newcomer R, Koppes R, McLean SG (2008) Combined effects of fatigue and decision making on female lower limb landing postures: central and peripheral contributions to ACL injury risk. Clin Biomech 23:81-92

11. Cohen J (1988) Statistical power analysis for the behavioral sciences. Lawrence Erlbaum Associate, Hillsdale

12. Czuppon S, Racette BA, Klein SE, Harris-Hayes M (2014) Variables associated with return to sport following anterior cruciate ligament reconstruction: a systematic review. Br J Sports Med 48(5):356-364

13. Decker LM, Moraiti C, Stergiou N et al (2011) New insights into anterior cruciate ligament deficiency and reconstruction through the assessment of knee kinematic variability in terms of nonlinear dynamics. Knee Surg Sports Traumatol Arthrosc 19:1620-1633

14. Dingenen B, Gokeler A (2017) Optimization of the return-to-sport paradigm after anterior cruciate ligament reconstruction: a critical step back to move forward. Sports Med 47(8):1487-1500
15. Ebert JR, Edwards P, Yi L, Joss B, Ackland T, Carey-Smith R et al (2017) Strength and functional symmetry is associated with post-operative rehabilitation in patients following anterior cruciate ligament reconstruction. Knee Surg Sports Traumatol Arthrosc. https://doi.org/10.1007/s00167-017-4712-6

16. Feucht MJ, Cotic M, Saier T, Minzlaff P, Plath JE, Imhoff AB et al (2016) Patient expectations of primary and revision anterior cruciate ligament reconstruction. Knee Surg Sports Traumatol Arthrosc 24:201-207

17. Gokeler A, Welling W, Benjaminse A, Lemmink K, Seil R, Zaffagnini S (2017) A critical analysis of limb symmetry indices of hop tests in athletes after anterior cruciate ligament reconstruction: A case control study. Orthop Traumatol Surg Res 103(6):947-951

18. Gokeler A, Welling W, Zaffagnini S, Seil R, Padua D (2017) Development of a test battery to enhance safe return to sports after anterior cruciate ligament reconstruction. Knee Surg Sports Traumatol Arthrosc 25(1):192-199

19. Grevnerts HT, Terwee CB, Kvist J (2015) The measurement properties of the IKDC-subjective knee form. Knee Surg Sports Traumatol Arthrosc 23(12):3698-3706

20. Grindem H, Logerstedt D, Eitzen I, Moksnes H, Axe MJ, Snyder-Mackler L et al (2011) Single-legged hop tests as predictors of self-reported knee function in nonoperatively treated individuals with anterior cruciate ligament injury. Am J Sports Med 39(11):2347-2354

21. Grindem H, Snyder-Mackler L, Moksnes H, Engebretsen L, Risberg MA (2016) Simple decision rules can reduce reinjury risk by $84 \%$ after ACL reconstruction: the Delaware-Oslo ACL cohort study. Br J Sports Med. https://doi.org/10.1136/bjsports-2016096031

22. Hewett TE, Myer GD, Zazulak BT (2008) Hamstrings to quadriceps peak torque ratios diverge between sexes with increasing isokinetic angular velocity. J Sci Med Sport 11(5):452-459

23. Kean CO, Birmingham TB, Garland SJ, Bryant DM, Giffin JR (2010) Minimal detectable change in quadriceps strength and voluntary muscle activation in patients with knee osteoarthritis. Arch Phys Med Rehabil 91(9):1447-1451

24. Kockum B, Heijne AI (2015) Hop performance and leg muscle power in athletes: reliability of a test battery. Phys Ther Sport 16(3):222-227

25. Kuenze C, Hertel J, Saliba S, Diduch DR, Weltman A, Hart JM (2015) Clinical thresholds for quadriceps assessment after anterior cruciate ligament reconstruction. J Sport Rehab 24(1):36-46

26. Kyritsis P, Bahr R, Landreau P, Miladi R, Witvrouw E (2016) Likelihood of ACL graft rupture: not meeting six clinical discharge criteria before return to sport is associated with a four times greater risk of rupture. Br J Sports Med 50:946-951

27. Logerstedt D, Di Stasi S, Grindem H, Lynch A, Eitzen I, Engebretsen L, Risberg MA, Axe MJ, Snyder-Mackler L (2014) Selfreported knee function can identify athletes who fail return to activity criteria up to 1 year after anterior cruciate ligament reconstruction: a Delaware-Oslo ACL cohort study. J Orthop Sports Phys Ther 44(12):914-923

28. Machado F, Debieux P, Kaleka CC, Astur D, Peccin MS, Cohen M (2018) Knee isokinetic performance following anterior cruciate ligament reconstruction: patellar tendon versus hamstrings graft. Phys Sports Med 46(1):30-35

29. Munro AG, Herrington LC (2011) Between-session reliability of four hop tests and the agility T-test. J Strength Cond Res 25(5):1470-1477

30. Myers BA, Jenkins WL, Killian C, Rundquist P (2014) Normative data for hop tests in high school and collegiate basketball and soccer players. Int J Sports Phys Ther 9:596-603 
31. Noyes FR, Barber SD, Mangine RE (1991) Abnormal lower limb symmetry determined by function hop tests after anterior cruciate ligament rupture. Am J Sports Med 19(5):513-518

32. Padua DA, Boling MC, Distefano LJ, Onate JA, Beutler AI, Marshall SW (2011) Reliability of the landing error scoring systemreal time, a clinical assessment tool of jump-landing biomechanics. J Sport Rehabil 20(2):145-156

33. Padua DA, Marshall SW, Boling MC, Thigpen CA, Garrett WE Jr, Beutler AI (2009) The Landing Error Scoring System (LESS) is a valid and reliable clinical assessment tool of jump landing biomechanics: the JUMP-ACL study. Am J Sports Med 37(10):1996-2002

34. Paterno MV, Rauh MJ, Schmitt LC, Ford KR, Hewett TE (2014) Incidence of second ACL injuries 2 years after primary ACL reconstruction and return to sport. Am J Sports Med 42:1567-1573

35. Santamaria LJ, Webster KE (2010) The effect of fatigue on lowerlimb biomechanics during single-limb landings: a systematic review. J Orthop Sports Phys Ther 40:464-473

36. Thomee R, Kaplan Y, Kvist J, Myklebust G, Risberg MA, Theisen D, Tsepis E, Werner S, Wondrasch B, Witvrouw E (2011) Muscle strength and hop performance criteria prior to return to sports after ACL reconstruction. Knee Surg Sports Traumatol Arthrosc 19(11):1798-1805

37. Thomee R, Neeter C, Gustavsson A, Thomee P, Augustsson J, Eriksson B, Karlsson J (2012) Variability in leg muscle power and hop performance after anterior cruciate ligament reconstruction. Knee Surg. Sports Traumatol Arthrosc 20(6):1143-1151

38. Tiffreau V, Ledoux I, Eymard B, Thevenon A, Hogrel JY (2007) Isokinetic muscle testing for weak patients suffering from neuromuscular disorders: a reliability study. Neuromuscul Disord 17(7):524-531

39. Toole AR, Ithurburn MP, Rauh MJ, Hewett TE, Paterno MV, Schmitt LC (2017) Young athletes after anterior cruciate ligament reconstruction cleared for sports participation: how many actually meet recommended return-to-sport criteria cutoffs? J Orthop Sports Phys Ther 47(11):825-833

40. Reid A, Birmingham TB, Stratford PW, Alcock GK, Giffin JR (2007) Hop testing provides a reliable and valid outcome measure during rehabilitation after anterior cruciate ligament reconstruction. Phys Ther 87(3):337-349

41. Van Melick N, van Cingel REH, Brooijmans F et al (2016) Evidence-based clinical practice update: practice guidelines for anterior cruciate ligament rehabilitation based on systematic review and multidisciplinary consensus. Br J Sports Med 50(24):1506-1515

42. Wellsandt E, Failla MJ, Snyder-Mackler L (2017) Limb symmetry indexes can overestimate knee function after anterior cruciate ligament injury. J Orthop Sports Phys Ther 47(5):334-338

43. Wiggins AJ, Grandhi RK, Schneider DK, Stanfield D, Webster KE, Myer GD (2016) Risk of secondary injury in younger athletes after anterior cruciate ligament reconstruction: a systematic review and meta-analysis. Am J Sports Med 44(7):1861-1876

44. Wilk KE (2015) Anterior cruciate ligament injury prevention and rehabilitation: let's get it right. J Orthop Sports Phys Ther 45(10):729-730

45. Zaffagnini S, Grassi A, Marcheggiani Muccioli GM, Tsapralis K, Ricci M, Bragonzoni L et al (2014) Return to sport after anterior cruciate ligament reconstruction in professional soccer players. Knee 21:731-735 